\title{
Evaluating Compositional Variation in InGaAs Random Alloys Using Atom Probe Tomography
}

Nicole A. Kotulak ${ }^{1}$, Mark E. Twigg ${ }^{1}$, Nadeemullah A. Mahadik ${ }^{1}$, Stephanie Tomasulo ${ }^{1}$, P. L. Bonanno ${ }^{2}$, N. R. Pereira ${ }^{3}$, Keith Knipling ${ }^{1}$, Michael K. Yakes ${ }^{1}$

1. U.S. Naval Research Laboratory, Washington, DC, USA

2. ASEE Postdoctoral Associate residing at the U.S. Naval Research Laboratory

${ }^{3 .}$ Ecopulse, Inc., Springfield, VA, USA

Highly engineered semiconductor devices, especially those implementing III-V random alloys, require materials that are equally as engineered. Precise control of the mechanisms involved in material growth can have a direct impact on the optoelectronic properties of these materials, ultimately affecting the performance of the device in which they are being implemented. We have recently evaluated InAlAsSb and found that its predicted optoelectronic properties did not match actual performance values. In that study, the InAlAsSb grown lattice-matched to InP exhibited narrower bandgaps than those predicted, with photoluminescence indicating the presence of both type II band alignment and carrier confinement. While secondary ion mass spectrometry (SIMS) and X-ray diffraction (XRD) confirmed that the bulk composition and lattice-matching were within measurement error of the target values [1], [2], transmission electron microscopy (TEM) and atom probe tomography (APT) indicated the existence of cluster features most likely on a scale of tens of angstroms [3], [4], [5], [6], [7].

In order to further investigate the growth kinetics of III-V random alloys, we have chosen to study InGaAs, a well-utilized model system that has demonstrated material degradation as a result of phase separation during growth [8]. Moreover, investigating a mixed group III ternary alloy reduces the complexity of analysis as only the group III elements should be competing for lattice sites. In this study, we present APT analysis in conjunction with TEM, grazing angle XRD, and $2 \mathrm{~d}$ small angle $\mathrm{x}$-ray scattering (SAXS) as a comparative materials characterization suite to confirm the existence of compositional variation in the film, identifying the size, distribution, and nature of these regions. The result is a better understanding of the growth kinetics for III-V alloy systems, with the goal of allowing future complex growth challenges to be addressed.

A Riber compact 21DZ molecular beam epitaxy (MBE) system was used to grow the InGaAs alloy samples investigated in this work. The InGaAs was grown at a substrate temperature $\left(\mathrm{T}_{\text {sub }}\right)$ of $400^{\circ} \mathrm{C}$ (measured by thermocouple), lattice-matched to the $\operatorname{InP}(100)$ substrate. The orientation of the substrate included a $2^{\circ}$ offcut, with the direction of the offcut along a line $45^{\circ}$ away from both [011] and [01-1]. The $\mathrm{T}_{\text {sub }}$ for growth is non-optimal for standard InGaAs growth recipes, so as to artificially induce/enhance and compositional variation in the material. 2d-SAXS measurements were performed at the Argonne National Laboratory 1BM beamline over the entire sample area. For APT analysis, an FEI Nova 600 NanoLab DualBeam ${ }^{T M}$ FIB/SEM was used to prepare specimens, including liftout, mounting onto flat-topped Si microtip arrays, and final shaping. APT data collection was performed using a Cameca 4000X Si LEAP, with a specimen temperature of $25 \mathrm{~K}, 0.01 \mathrm{pJ}$ nominal laser pulse energy, 125 $\mathrm{kHz}$ pulse repetition rate, and a $0.5 \%$ detection rate. Cameca Integrated Visualization and Analysis Software (IVAS) 3.8.0 was used for reconstruction and analysis.

After $3 \mathrm{D}$ reconstruction, a $20 \times 20 \times 55 \mathrm{~nm}^{3}$ region of interest (ROI), with the z-axis parallel to the 
growth direction, was selected within the InGaAs film for further compositional analysis. A 2D composition contour plot, using voxel size of $0.5 \mathrm{~nm} / \mathrm{side}$, for each species of interest was created perpendicular to the growth direction (Fig. 1a). These plots show correlation between high/low In and low/high Ga. A 1D composition profile through the ROI, running parallel to the growth direction, exhibits periodic fluctuation in composition along the growth direction (Fig. 1b). The length-scale of the periods ranges from $4 \mathrm{~nm}$ to $10 \mathrm{~nm}$, with an average length of $6.2 \mathrm{~nm}$ per period within the analyzed region. Dark-field cross-sectional TEM (XTEM) imaging reveals a wavelength of $8 \mathrm{~nm}$ along the [001] growth direction (Fig. 1c). Grazing angle XRD with spot size of $\sim 5 \times 5 \mathrm{~mm}^{2}$ shows interference fringes with a double peak period of $\sim 20 \mathrm{~nm}$, indicating vertical segregation present throughout the sample. The 2d-SAXS measurements show the presence of a double period in the interference fringes for the sample grown at $400^{\circ} \mathrm{C}$ with $8.1 \mathrm{~nm}$ thick layers (Fig. 1d). [9]

References:

[1] M. P. Lumb, et al., Proceedings of SPIE (2012) p. 8256.

[2] L. C. Hirst, et al., J. Appl. Phys. 117 (2015), p. 215704.

[3] L. C. Hirst, et al., ACS Nano 11(3) (2017), p. 2734.

[4] N. A. Kotulak, et al., Microsc. Microanal. 23(Suppl 1) (2017), p. 718.

[5] N. A. Kotulak, et al., Proceedings of $43^{\text {rd }}$ IEEE PVSC (2016).

[6] N. Baladés, et al., Applied Surface Science 395 (2017), p. 98.

[7] J. Hernández-Saz, et al., Nanotechnology 27 (2016), p. 305402.

[8] F. Peiro et al., J. Appl. Phys. 73 (1993), p. 4913.

[9] The authors acknowledge funding support from the Office of Naval Research. P. L. B. held an ASEE Postdoctoral Fellowship at the time this work was performed.

a)

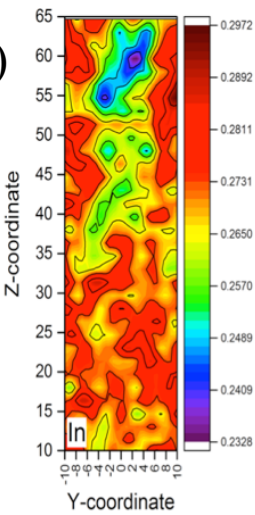

c)

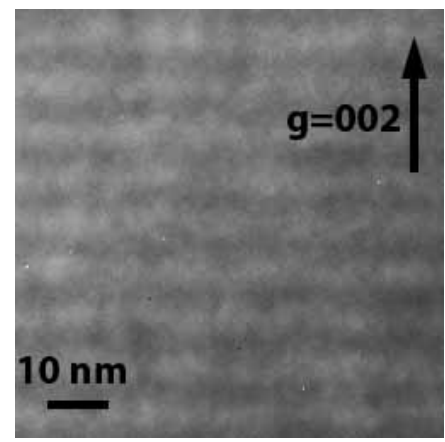

b)

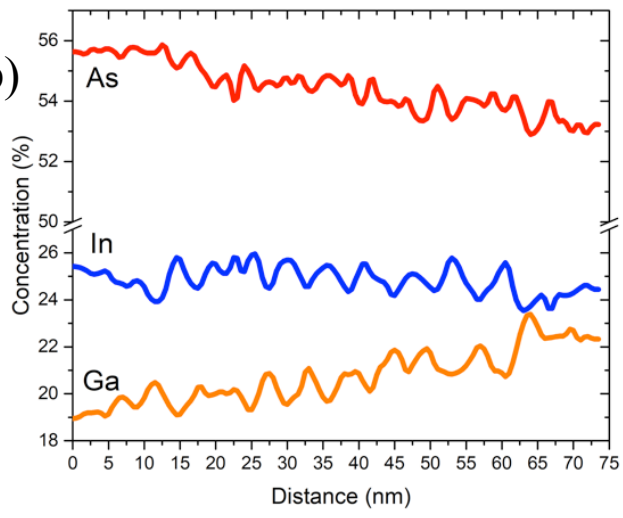

d)

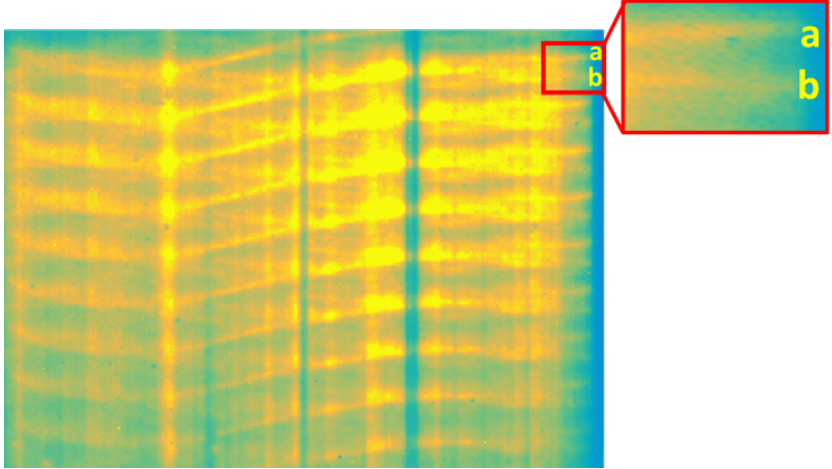

Figure 1. a) 2D composition contour plots for a $20 \mathrm{~nm}$ thick slice parallel to the growth/analysis direction, b) 1D composition profile along the growth/analysis direction, c) Dark-field XTEM image of phase separation in InGaAs film, d) 2d-SAXS image of InGaAs grown at $\mathrm{T}_{\text {sub }}=400^{\circ} \mathrm{C}$. 